\title{
Field evaluation of a recombinase polymerase amplification assay for the diagnosis of Schistosoma japonicum infection in Hunan province of China
}

Weiwei Xing ${ }^{1}$, Xinling $\mathrm{Yu}^{2}$, Jingtao Feng ${ }^{2}$, Kui Sun ${ }^{1}$, Wenliang Fu${ }^{1}$, Yuanyuan Wang ${ }^{1}$, Minji Zou', Wenrong Xia', Zhihong LuO ${ }^{2}$, Hongbin $\mathrm{He}^{2}$, Yuesheng $\mathrm{Li}^{3}$ and Donggang $\mathrm{Xu}^{{ }^{* *}}$

\begin{abstract}
Background: Current diagnostic methods for Schistosoma japonicum infection are insensitive for low-density infections. Therefore, a new diagnostic assay based on recombinase polymerase amplification (RPA) technology was established and assessed for field applification.

Methods: The S.japonicum RPA assay was developed to target highly repetitive retrotransposon SjR2 gene of S japonicum, and its sensitivity and specificity were assessed by serial dilution of S. japonicum genomic DNA and other related worm genomic DNA respectively. The RPA diagnostic validity was first evaluated in 60 fecal samples from healthy people and patients, and then compared with other diagnostic tests in 200 high-risk individuals living in endemic areas.

Results: The real time RPA assay could detect $0.9 \mathrm{fg}$ S. japonicum DNA within 15 min and distinguish S. japonicum from other worms. The validity analysis of RPA for the detection of S. japonicum in stool samples from 30 S. japonicum-infected patients and 30 healthy persons indicated $100 \%$ sensitivity and specificity. When testing 200 fecal or serum samples from a high-risk population, the percentage sensitivity of RPA was 100\%, whereas that of indirect hemagglutination assay (IHA) and enzyme-linked immunosorbent assay (ELISA) were $80.3 \%$ and $85.2 \%$ respectively. In addition, the RPA presented better consistency with the stool-based tests than IHA and ELISA. Overall, the RPA was superior to other detection methods with respect to detection time, sensitivity, and convenience.
\end{abstract}

Conclusions: This is the first time we applied the RPA technology to the field evaluation of S. japonicum infection. And the results suggest that RPA-based assays can be used as a promising point-of-care test for the diagnosis of schistosomiasis.

Keywords: Schistosoma japonicum, Recombinase polymerase amplification, Field evaluation, Diagnosis

\section{Background}

Schistosomiasis japonica is a major tropical disease in China, with a $>2100$-year documented history [1]. With the implementation of the National Control Program supported by the Chinese government, China has made great progress in reducing S. japonicum infections in humans [2-5]. Today, the prevalence is becoming more

\footnotetext{
*Correspondence: xudg@bmi.ac.cn

${ }^{1}$ The Laboratory of genomic engineering, Beijing Institute of Basic Medical Sciences, Beijing, China

Full list of author information is available at the end of the article
}

and more low in most of the endemic areas. Under such circumstances, current diagnostic methods became less sensitive and specific which make the control program in a difficult situation. Generally, S. japonicum infections are diagnosed by direct parasitological methods or immunological methods. Parasitological detections, including the Kato-Katz (KK) thick smear and the miracidium hatching test (MHT), were regarded as golden standard for the diagnosis of schistosomiasis. However, parasitological detection is labor-intensive, time-consuming, and exhibits low sensitivity, which is not suitable for 
large-scale disease surveillance [6, 7]. Immunological methods include indirect hemagglutination assay (IHA) and enzyme-linked immunosorbent assay (ELISA). Both of them are more sensitive and convenient than parasitological methods. However, the above immunologic detection methods are usually not species-specific and can not discriminate between the active and past $S$. japonicum infection. Many studies demonstrated that false-positive rates of IHA and ELISA were very high in field settings [8-11]. Recently, Pan et al. verified a potential protein marker, SjSP-13, using genome-wide methods, and the SjSP-13-based ELISA kit showing 90.4\% sensitivity and $98.9 \%$ specificity in a field study. However, its validity still needs further confirmation in large-scale population studies [12]. Given that the currently available diagnostic methods are not very satisfactory, development and evaluation of new strategies and tools for the control of schistosomiasis were recommended by the World Health Organization [13]. With the development of nucleic amplification technology, polymerase chain reaction (PCR) and other isothermal amplification technologies have been described for the diagnosis of schistosomiasis [14, 15]. Although PCR-based assays provide sensitive, specific and reliable tools, they are not widely utilized due to the dependence on expensive apparatus and training operator, which limits their large-scale application for clinical diagnosis [16].

In 2006, Piepenburg et al introduced a novel isothermal technology called recombinase polymerase amplification (RPA) for molecular diagnosis [17]. Unlike many other amplification methods, RPA does not require thermal denaturation of template but utilizes recombinase enzyme with opposing oligonucleotide primers to scan duplex DNA and facilitate strand exchange at cognate sites. The reaction progresses rapidly and results in specific DNA amplification from just a few target copies to detectable levels typically at temperatures between $25{ }^{\circ} \mathrm{C}$ and $42{ }^{\circ} \mathrm{C}$. With RPA probes which contain a specific abasic nucleotide analogue flanked by a dT-fluorophore and a corresponding dT-quencher group, we can monitor amplication events in the reaction [17]. Since RPA has advantages, including a broad range of incubation temperatures $\left(25-42{ }^{\circ} \mathrm{C}\right)$, shorter reaction times (typically $<15 \mathrm{~min}$ ), and more flexibility in basic laboratory settings in the field, it has gained further attention in point-of-care testing.

Here, we developed a real-time RPA assay for rapid detection of $S$. japonicum DNA in fecal samples and compared this assay with current methods in terms of sensitivity and specificity for the diagnosis of S. japonicum infection in high-risk populations.

\section{Methods}

\section{RPA primer and probe}

The highly repetitive retrotransposon $\mathrm{SjR} 2$ of $S$. japonicum (GenBank accession No. AF412221) was used for DNA detection as a target sequence [18-20]. The SjR2-specific primers and probe for RPA were designed according to Piepenburg [17], and the optimal combination of primers and probe were shown in Table 1. All oligonucleotides were produced by Sangon Biotech, Beijing, China.

\section{DNA extraction}

Samples of genomic DNA were extracted from the adult worms of S. japonicum, S. haematobium, S. mansoni, and $S$. sinensium by a DNeasy Tissue Kit (Qiagen, CA, USA) following the manufacturer's instruction. The concentration and purity of the DNA were determined spectrophotometrically by readings at wavelengths of $260 \mathrm{~nm}$ and $280 \mathrm{~nm}$ (Eppendorf BioSpectrometer, Hamburg, Germany).

\section{RPA reactions}

RPA was performed in a total volume of $50 \mu \mathrm{L}$, using a TwistAmp Exo kit (TwistDX Ltd., Cambridge, UK). Each reaction contained $29.5 \mu \mathrm{l}$ TwistAmp rehydration buffer, $2.1 \mu \mathrm{l}$ each RPA primer $(210 \mathrm{nM}), 0.6 \mu \mathrm{l}$ RPA probe $(120 \mathrm{nM}), 12.2 \mu \mathrm{l}$ nuclease-free water, $1 \mu \mathrm{l}$ template and $2.5 \mu \mathrm{l}$ magnesium acetate. All reagents except for the magnesium acetate and template DNA were pipette into a $0.2 \mathrm{ml}$ reaction tube which contains a dried enzyme pellet. To start the reaction magnesium acetate and template DNA were added. Then the tube was placed in the Twista ${ }^{\mathrm{TM}}$ incubator block $\left(39^{\circ} \mathrm{C}\right)$ and the fluorescence measurement was initiated to minitor the progression of RPA reactions. The preincubation was performed for $1 \mathrm{~min}$ and followed by incubation for $20 \mathrm{~min}$, with brief mixing of the mixtures after a 4 min incubation step. In combination with the nuclease sensitive fluorophore/ quencher probes, a real-time DNA detection system was constituted. The labeled amplicon that generated in the reaction can be measured via 6-carboxyfluorescein (FAM) fluorescence using the Twista ${ }^{\text {TM }}$ reader every $20 \mathrm{~s}$.

Table 1 RPA primers and probe designed in this study

\begin{tabular}{ll}
\hline Name & Sequence \\
\hline S. japonicum RPA FP & CCAAGTCTCAGTGAAGTTGTGAAGGCTAT \\
S. japonicum RPA RP & GTTAGTGTTCGAGACCAGTCAGATGGGATT \\
S. japonicum RPA P & CTTAAAGCGAGGGAGAGCGGCAGGACCAGA \\
& (dT-FAM)G(THF)A(dT-BHQ1)TGACCCCTG \\
& AGATAT[3'-block] \\
\hline
\end{tabular}

$F P$ forward primer, $R P$ reverse primers, $P$ probe, $d T-F A M$ thymidine nucleotide carrying fluorescein, $T H F$ tetrahydrofuran spacer, $d T-B H Q 1$ thymidine nucleotide carrying black hole quencher 
The RPA fluorescence data were assessed by taking a baseline relative fluorescence unit measurement.

\section{Real-time PCR}

Real-time PCR was performed using SYBR Green Mastermix (Fermentas, Ontario, Canada) and the ABI 7500 QPCR system according to manufacturer instructions. All samples were processed in triplicate. Primers used in amplifications were as follows: SJR2: 5'-GAC AGG TTC TGG AAC ATA GG-3'; SJR2: 5' -GGT CAA TTC CGA AGA CAA TC3'.

\section{Sensitivity and specificity tests}

The sensitivity of the RPA-assay was evaluated using five 10 -fold serial dilutions ranging from $9 \mathrm{pg} / \mu \mathrm{L}$ to $0.9 \mathrm{fg} /$ $\mu \mathrm{L}$ genomic DNA extracted from an S. japonicum worm. The specificity of detection was performed using genomic DNA from S. japonicum, S. haematobium, S. mansoni, and S. sinensium.

\section{Analysis of RPA diagnostic validity}

Thirty infected fecal samples collected from Xiangyue Hospital in Yueyang city were diagnosed by the KK method as egg-positive. The other thirty uninfected fecal samples were collected from health volunteers living in the Haidian District of Beijing, where schistosomiasis is not endemic. We then used RPA technology to analyze the fecal samples and assess the sensitivity and specificity of the RPA system.

\section{Field evaluation}

Two-hundred fishermen living in Yueyang County near Dongting Lake in the northeastern region of Hunan Province were enrolled in the field study to examine the potential diagnostic value. The inhabitants became infected with S. japonicum owing to frequent agricultural activities and fishing in snail-infested marshlands.. Total DNA from $500 \mathrm{mg}$ of fecal sample of each participant was extracted using the QIAamp DNA Stool Mini Kit (Qiagen GmbH, Hilden, Germany) according to manufacturer instructions. The other fecal sample from each participant was analyzed with MHT and KK. $3 \mathrm{~mL}$ blood sample was collected from each participant and was centrifuged as $760 \times \mathrm{g}$ at $4{ }^{\circ} \mathrm{C}$ for $10 \mathrm{~min}$. The obtained serum samples were stored as $-80{ }^{\circ} \mathrm{C}$ for ELISA and IHA measurement.

\section{MHT and KK thick smear}

The MHT was performed by adding about $30 \mathrm{~g}$ of feces into a metal container with a coarse wire mesh (150 holes per square inch). With the flowing water and a stirring stick, fine material was flushed into a 300-mesh nylon bag and flushed with water until the sediment was clear. Then the sediment was collected and transferred to a triangular flask containing $300 \mathrm{~mL}$ non-chlorinated water from the Yangtze River. Finally, the flasks were left in a well-lit room with the temperature set at approximately $28{ }^{\circ} \mathrm{C}$ and examined with a magnifying glass to check the present of swimming miracidia after 4, 6, 8, and $24 \mathrm{~h}$.

The KK thick smear was performed according to a standardized methodology [21]. The method based on three slides by using fresh stool specimen, nylon screens and plastic templates (Zhejiang Ningbo Medical Instrument Factory, Ningbo, China). The three slides (41.7 mg each) were prepared from one stool sample and examined within 1 week. The intensity of infection was calculated by multiplying the number of eggs per glass slide by 24 .

\section{ELISA and IHA}

S. japonicum IgG ELISA kit (BIOCBD, Shenzhen, China) was used to detect the infection status of the high-risk population. All procedures were conducted according to manufacturer instructions. The IHA detection kit was purchased from Anhui Provincial Institute of Parasitic Diseases (Wuhu, China). The testing procedure was performed as described by Zhou et al [8]. The test result was recognized to be positive when a positive reaction appeared at an antibody titer of $\geq 1: 10$.

\section{Statistical analysis}

In the field survey, the combination of the KK method and the MHT was considered the diagnostic gold standard, and the diagnostic performance of RPA was assessed by calculating sensitivity, specificity, positive predictive value (PPV), and negative predictive value (NPV). The indexes were calculated using SAS 9.2 software (SAS Institute, Inc., Cary, NC, USA), and agreement statistics were determined (JMP v9, SAS Institute Inc.) to establish the agreement between two diagnostic tests. A $p<0.05$ was considered significant.

\section{Results}

\section{Sensitivity and specificity of the RPA assay for S. japonicum}

The analytical sensitivity of the assay was evaluated with 10 -fold serial dilutions ranging from $9 \mathrm{pg} / \mu \mathrm{L}$ to $0.9 \mathrm{fg} /$ $\mu \mathrm{L}$ of genomic DNA extracted from an S. japonicum worm. The results showed that the RPA limit of detection was 0.9 fg DNA per reaction, which is equal to that of real-time PCR (Fig. 1a and b). Although the RPA sensitivity was equal to real-time PCR, the former was far superior to the latter in detection time and convenience. RPA specificity for $S$. japonicum was confirmed by cross-detection assays using the genomic DNA of $S$. mansoni, S. sinensium, and S. haematobium, while none of these was amplified (Fig. 2). 

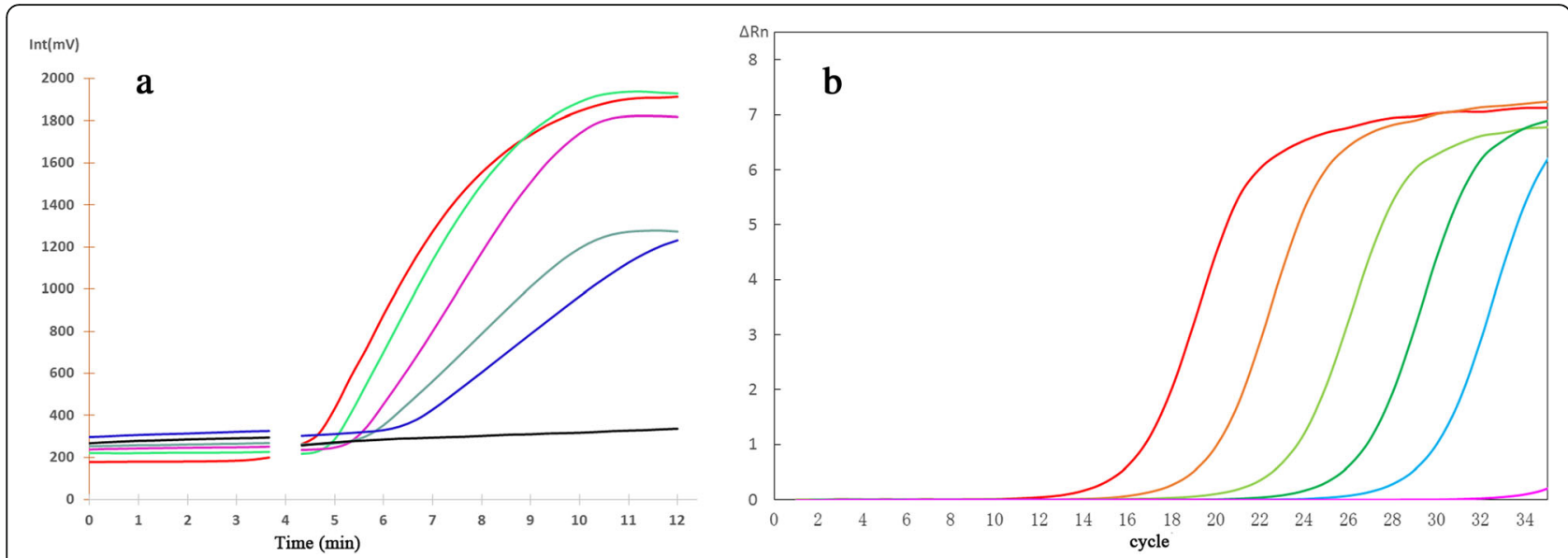

Fig 1 Analytical sensitivity of real-time RPA and real-time PCR for Schistosoma japonicum detection. Fluorescence development via real-time detection using a dilution range of $9 \mathrm{pg} / \mu \mathrm{L}-0.9 \mathrm{fg} / \mu \mathrm{L}$ of the $S$. japonicum genomic DNA. a Real-time RPA: $9 \mathrm{pg} / \mu \mathrm{L}$, represented by the red line; $900 \mathrm{fg} / \mu \mathrm{L}$, green; $90 \mathrm{fg} / \mu \mathrm{L}$, pink; $9 \mathrm{fg} / \mu \mathrm{L}$, cyan; $0.9 \mathrm{fg} / \mu \mathrm{L}$, blue; negative control, black. b Real-time PCR: $9 \mathrm{pg} / \mu \mathrm{L}$ represented by the red line; $900 \mathrm{fg} / \mu \mathrm{L}$, orange; $90 \mathrm{fg} / \mu \mathrm{L}$, light green; $9 \mathrm{fg} / \mu \mathrm{L}$, green; $0.9 \mathrm{fg} / \mu \mathrm{L}$, cyan; negative control, pink

\section{Analysis of RPA diagnostic validity}

We analyzed RPA diagnostic validity using 30 infected and 30 uninfected fecal samples. Compared to KK method, RPA diagnostic sensitivity and specificity were both $100 \%$ for stool samples (Table 2). This result indicated that the RPA method was valid for S. japonicum diagnosis.

\section{Comparison of the RPA assay, ELISA, IHA, MHT and the modified KK method in terms of sensitivity and specificity for the detection of S. japonicum infection in the field study}

To further assess the RPA assay, we performed a field study to evaluate its sensitivity and specificity. The RPA diagnostic validity was compared with that of ELISA, IHA, the MHT and the modified KK method. Two-hundred inhabitants were enrolled in the study, including 92 male and 108 female participants. Among these, 164 (82\%) were aged $15-54$ years, and 36 (18\%) were aged 55-78 years, and the local fishermen were assessed between March 2013 and April 2014. Of the 200 enrolled residents, 61 (31.5\%) individuals were identified as being positive using the MHT, which included 48 (24\%) individuals tested positive using the KK method. All individuals diagnosed as positive for parasitological infection were also diagnosed as being positive by RPA detection (Fig. 3). In conclusion, the RPA identified more positive cases than either, including samples negative by the other techniques (Table 3). Here, the combination of the KK and MHT methods was considered the diagnostic gold standard. The sensitivity and specificity of ELISA and IHA were $85.2 \%$ and $93.5 \%, 80.3 \%$ and $83.4 \%$ respectively, while RPA sensitivity and specificity was $100 \%$

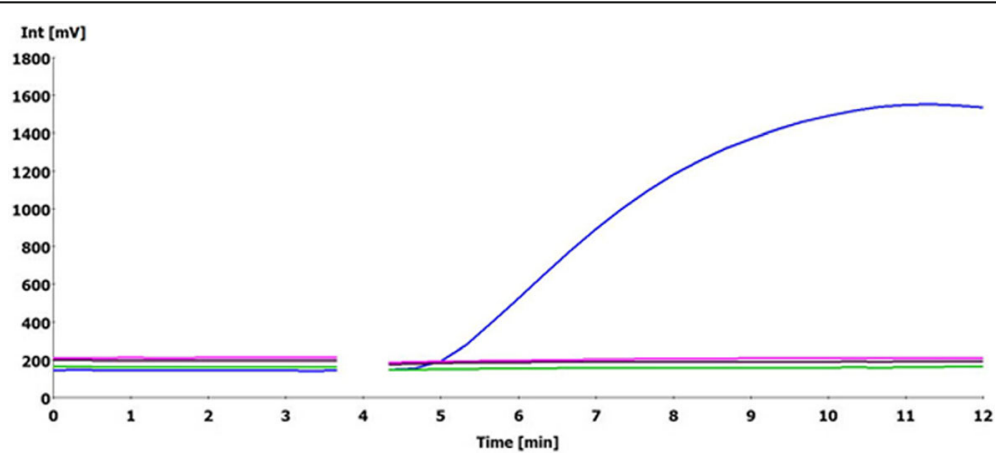

Fig 2 The specificity of Schistosoma japonicum RPA. S. japonicum is represented by the blue line; S. mansoni, black; S. sinensium, green; S. haematobium, pink 
Table 2 Analysis of diagnostic validity of RPA

\begin{tabular}{lll}
\hline Subjects & Investigated & RPA-positive \\
\hline Controls (negative by KK method) & 30 & $0(0 \%)$ \\
Cases (positive by KK method) & 30 & $30(100 \%)$ \\
\hline
\end{tabular}

and $96.4 \%$, respectively (Table 3 ). In addition, the RPA showed a significantly higher degree of agreement with the gold standards (kappa: 0.942; 95\% confidence interval (CI): $0.89-0.99 ; p<0.001)$ relative to IHA and ELISA [IHA kappa: 0.61 (95\% CI: 0.49-0.72), $p<0.001$; ELISA kappa: 0.78 (95\% CI: 0.69-0.88), $p<0.001$ ] (Table 4).

\section{Discussion}

The global schistosomiasis control program has made tremendous progress in reducing the prevalence of the disease and morbidity in many endemic areas, particularly in China [22]. However, the complete elimination of schistosomiasis associated with $S$. japonicum and the prevention of its reemergence remain difficult due to the limitations of detection methods [23]. Therefore, it is necessary to develop an efficient and convenient detection method for overall control of schistosomiasis infection.

Recently, related DNA-amplification technologies, including PCR, real-time PCR, and isothermal nucleic acid amplification, were developed to detect $S$. japonicum infections and showed potential as highly sensitive and specific techniques for the detection of parasite DNA in feces or sera, especially in regions with low-intensity infections. However, PCR and real-time PCR were extremely dependent on a well-equipped laboratory,

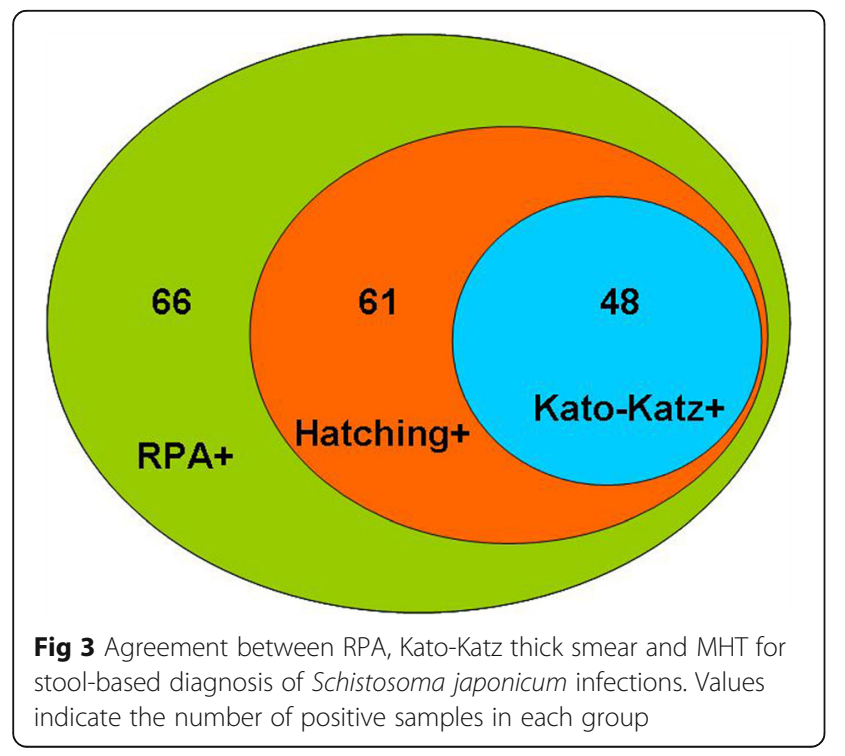

given that testing times usually take 60-90 min, and the equipment is heavy, expensive, complex, and must be operated by qualified staff. Additionally, the drawbacks of isothermal amplification methods such as LAMP are as follows: the design of appropriate LAMP primers is more complicated than that of PCR and RPA primers, and LAMP requires higher incubation temperatures ranging from $55{ }^{\circ} \mathrm{C}$ to $65{ }^{\circ} \mathrm{C}$ for 60-75 min, with the amplification products difficult to quantify [24].

In this study, RPA technology was used to detect S. japonicum infection, with the diagnostic method revealing substantial advantages over current methods. First, RPA showed adequate sensitivity and specificity required for identification of low-intensity infections. The RPA limit of detection reached $0.9 \mathrm{fg}$ per reaction, which was equal to real-time PCR. The non-long terminal repeat (LTR) retrotransposons (SjR2) was chose as detection targets, which were highly conserved and repeatable in the S. japonicum genome [20]. And we found there was no crossreactivity with other fluke infections. Second, the RPA technique combined with the fluorescent probe achieved detection within 10-15 min, which was a much shorter duration relative to that of current methods. Third, the RPA can be operated at a relative low and constant temperature and the only requirements are primers, the probe, and the sample, while others can be stored in lyophilized form, which facilitates field testing or point-of-care applications. The above advantages make RPA more suitable for the application in field study or point of care test. Recently a LFD-RPA assay which combine recombinase polymerase amplification and lateral flow dipstick was also developed. It adopted a lateral flow system to readout the results with naked eyes which seemed really rapid and applicable in field. Nevertheless, current LFD-RPA assay requires additional steps to transfer the amplified product to another opened tube for lateral flow detection, which may lead to the possibility of nucleic acid contamination $[25,26]$. Therefore, the LFD-RPA assay still needs to be further improved for practical application.

Further, the validity analysis for RPA in the detection of S. japonicum in the stool samples from 30 controls and 30 cases indicated that the method exhibited excellent sensitivity and specificity. Therefore, RPA technology was then applied in the field survey of $S$. japonica infection and compared with current detection methods. The field study showed that the RPA assay identified 66 enrolled participants as positive, which included all individuals diagnosed as positive by the MHT and KK methods. Among all the participants, RPA technique identified $33 \%$ of 
Table 3 Estimation of diagnostic validity of RPA, IHA and ELISA

\begin{tabular}{lllllllll}
\hline Methods & $\begin{array}{l}\text { TRUE } \\
\text { positive }\end{array}$ & $\begin{array}{l}\text { FALSE } \\
\text { negative }\end{array}$ & $\begin{array}{l}\text { TRUE } \\
\text { negative }\end{array}$ & $\begin{array}{l}\text { FALSE } \\
\text { positive }\end{array}$ & $\begin{array}{l}\text { Sensitivity } \\
\left(\%, 95 \% \mathrm{Cl}^{\mathrm{a}}\right)\end{array}$ & $\begin{array}{l}\text { Specificity } \\
\left(\%, 95 \% \mathrm{Cl}^{\mathrm{a}}\right)\end{array}$ & $\begin{array}{l}\text { PPV\% } \\
\left(95 \% \mathrm{Cl}^{\mathrm{a}}\right)\end{array}$ & $\begin{array}{l}\text { NPV\% } \\
\left(95 \% \mathrm{Cl}^{\mathrm{a}}\right)\end{array}$ \\
\hline RPA & 61 & 0 & 134 & 5 & $100 \%(100)$ & $96.40 \%(99.32-99.54)$ & $92.41 \%(86.06-98.83)$ & $100 \%(100)$ \\
IHA & 49 & 12 & 116 & 23 & $80.32 \%(70.37-90.45)$ & $83.45 \%(77.22-89.67)$ & $68.06 \%(57.37-78.82)$ & $90.67 \%(85.64-95.72)$ \\
ELISA & 52 & 9 & 130 & 9 & $85.24 \%(76.34-94.15)$ & $93.52 \%(89.44-97.61)$ & $85.37 \%(76.34-94.18)$ & $93.51 \%(89.45-97.67)$ \\
\hline
\end{tabular}

${ }^{\text {a }}$ Exact $95 \%$ confidence intervals (Cls)

infection intensity, while the KK method only identified only $24 \%$ of infection intensity. Furthermore, when the combination of the KK method and the MHT was regarded as the diagnostic golden standard,RPA sensitivity and specificity was $100 \%$ and $96.4 \%$, respectively, which was significantly superior to that of the IHA and ELISA methods. However, a significant drawback of RPA technology is its high cost per reaction. The primers and RPA exo reaction kits together cost approximately $\$ 4.3$, which was higher than current detection technology. Of course, with availability and throughput increase, the prices are likely to decrease in the future. Besides, some more simple and cheap DNA extraction methods are considering in our next field work, such as the commercially available magnetic bead-based strategy, noncommercial ROSE extraction method and heated $\mathrm{NaOH}$ method. All these considering methods are cheap and easy to perform which will greatly decrease the whole cost.

\section{Conclusion}

In this study, we developed an RPA assay for detection of S. japonicum and firstly applied it to epidemiological studies. Our results showed that the RPA assay was a very attractive nucleic acid detection method for the diagnosis of S. japonicum infection. This method exhibited high sensitivity, good specificity, convenient operation, minimal equipment requirement and rapid detection. And it could be applicable to not only schistosomiasis diagnosis but also environmental monitoring.

Table 4 Agreement statistics were calculated for RPA, IHA and ELISA with stool-based tests

\begin{tabular}{lccc}
\hline Diagnostic test & $\begin{array}{l}\text { Degree of agreement } \\
\text { - Kappa value }\end{array}$ & $95 \% \mathrm{Cl}^{\mathrm{a}}$ & $P$ value \\
\hline RPA vs. golden standard & 0.95 & $89-99$ & $<0.001$ \\
IHA vs. golden standard & 0.61 & $49-72$ & $<0.001$ \\
ELISA vs. golden standard & 0.78 & $69-88$ & $<0.001$ \\
\hline
\end{tabular}

${ }^{\mathrm{a}}$ Exact $95 \%$ confidence intervals (Cls)

\section{Abbreviations}

ELISA: Enzyme-linked immunosorbent assay; IHA: Indirect hemagglutination assay; KK: Kato-Katz thick smear; MHT: The miracidium hatching test; NPV: Negative predictive value; PPV: Positive predictive value; RPA: Recombinase polymerase amplification

\section{Acknowledgements}

Valuable thanks will be extended to the key laboratory of Immune and Control of Schistosomiasis, Human Institute of Parasitic Diseases for providing clinical infected specimens. We also thank all the study participants for their help.

\section{Funding}

This work was financially supported by the National Science and Technology Major Project of the Ministry of Science and Technology of China (grant number: 2013ZX10004101003 and 2012ZX10004909)

\section{Availability of data and materials}

The datasets generated and/or analysed during the current study are not publicly available due to participant privacy but are available from the corresponding author on reasonable request.

\section{Authors' contributions}

WWX and DGX were involved in conception, design, data collection and analysis and drafted the manuscript. JTF, KS, WLF, YYW, MJZ and WRX were involved in conception, design, data analysis and coordination of the study. XLY, ZHL, HBH, YSL were responsible for the sample collection, study design. All authors approved submission of the final manuscript.

\section{Competing interests}

The authors declare that they have no competing interests.

\section{Consent for publication}

Not applicable.

\section{Ethics and consent to participate}

All study procedures were conducted in accordance with and by approval of the internal review board of Beijing Institute of Basic Medical Sciences. The study was also approved by Hunan Institute of Parasitic Diseases. All participants received information on the aim and procedures of the study, and gave written informed consent.

\section{Author details}

${ }^{1}$ The Laboratory of genomic engineering, Beijing Institute of Basic Medical Sciences, Beijing, China. ${ }^{2}$ The key laboratory of Immune and Control of Schistosomiasis, Hunan Institute of Parasitic Diseases, Hunan, China.

${ }^{3}$ Queensland Institute of Medical Research, Brisbane, Australia.

Received: 17 June 2016 Accepted: 4 January 2017

Published online: 21 February 2017

\section{References}

1. Gryseels B, Polman K, Clerinx J, Kestens L. Human schistosomiasis. Lancet. 2006:368:1106-18.

2. Zhou XN, Wang LY, Chen MG, Wu XH, Jiang QW, Chen XY. The public health significance and control of schistosomiasis-then and now. Acta Trop. 2005;96:97-105. 
3. Mao SP, Shao BR. Schistosomiasis control in the People's Republic of China. Am J Trop Med Hyg. 1982;31:92-9.

4. Chen MG, Feng Z. Schistosomiasis control in China. Parasitol Int. 1999;48:11-9.

5. Yuan $\mathrm{HC}$, Jiang $\mathrm{QW}$, Zhao GM, He N. Achievements of schistosomiasis control in China. Mem Inst Oswaldo Cruz. 2002;97:187-9.

6. Lier T, Simonsen GS, Wang T, Lu D, Haukland HH, Vennervald BJ, et al. Realtime polymerase chain reaction for detection of low-intensity Schistosoma japonicum infections in China. Am J Trop Med Hyg. 2009;81:428-32.

7. Doenhoff MJ, Chiodini PL, Hamilton JV. Specific and sensitive diagnosis of schistosome infection: can it be done with antibodies? Trends Parasitol. 2004;20:35-9.

8. Zhou YB, Yang MX, Wang QZ, Zhao GM, Wei JG, Peng WX, et al. Field comparison of immunodiagnostic and parasitological techniques for the detection of Schistosomiasis japonica in the People's Republic of China. Am J Trop Med Hyg. 2007;76:1138-43.

9. Yu JM, de Vlas SJ, Jiang QW, Gryseels B. Comparison of the Kato-Katz technique, hatching test and indirect hemagglutination assay (IHA) for the diagnosis of Schistosoma japonicum infection in China. Parasitol Int. 2007; 56:45-9.

10. Wang QZ, Wang FF, Yin XM, et al. Evaluation of screening effects of ELISA and IHA techniques in different epidemic areas of schistosomiasis. J Trop Dis Parasitol. 2006;3:135-9. in Chinese.

11. Chen SR, Chen F, Zhou XN, Li HJ, Steinmann PJ, Yang Z. Comparison of aetiological and serological diagnosis methods in schistosomiasis mountainous endemic area. Parasitol Infect Dis. 2007;5:1-4. in Chinese.

12. Xu X, Zhang Y, Lin D, Zhang J, Xu J, Liu YM. Serodiagnosis of Schistosoma japonicum infection: genome-wide identification of a protein marker, and assessment of its diagnostic validity in a field study in China. Lancet Infect Dis. 2014;14:489-97.

13. WHO Expert Committee. Prevention and control of schistosomiasis and soiltransmitted helminthiasis. World Health Organ Tech Rep Ser. 2002;912:1-57.

14. Ibironke OA, Phillips AE, Garba A, Lamine SM, Schiff C. Diagnosis of Schistosoma haematobium by detection of specific DNA fragments from filtered urine samples. Am J Trop Med Hyg. 2011;84:998-1001.

15. Xu J, Rong R, Zhang HQ, Shi CJ, Xhu XQ, Xia CM. Sensitive and rapid detection of Schistosoma japonicum DNA by loop-mediated isothermal amplification (LAMP). Int J Parasitol. 2010;40:327-31.

16. Poschl B, Waneesorn J, Thekisoe O, Chutipongvivate S, Karanis P. Comparative diagnosis of malaria infections by microscopy nested PCR, and LAMP in northern Thailand. Am J Trop Med Hyg. 2010;83:56-60.

17. Piepenburg O, Williams $\mathrm{CH}$, Stemple DL, Armes NA. DNA detection using recombination proteins. PLoS Biol. 2006;4:e204.

18. Laha T, Brindley PJ, Smout MJ, Verity CK, McManus DP, Loukas A. Reverse transcriptase activity and untranslated region sharing of a new RTE-like, non-long terminal repeat retrotransposon from the human blood fluke, Schistosoma japonicum. Int J Parasitol. 2002;32:1163-74.

19. Wang $C$, Chen L, Yin X, Hua W, Hou M, Ji M, et al. Application of DNA-based diagnostics in detection of schistosomal DNA in early infection and after drug treatment. Parasit Vectors. 2011:4:164.

20. Guo JJ, Zheng HJ, Xu J, Xhu XQ, Wang SY, Xia CM. Sensitive and specific target sequences selected from retrotransposons of Schistosoma japonicum for the diagnosis of schistosomiasis. PLoS Negl Trop Dis. 2012;6:e1579.

21. Katz N, Chaves A, Pellegrino J. A simple device for quantitative stool thicksmear technique in Schistosomiasis mansoni. Rev Inst Med Trop Sao Paulo. 1972:14:397-400.

22. Chen MG. Assessment of morbidity due to Schistosoma japonicum infection in China. Infect Dis Poverty. 2014;3:6.

23. Zhou YB, Zheng HM, Jiang QW. A diagnostic challenge for Schistosomiasis japonicum in China: consequences on praziquantel-based morbidity control. Parasit Vectors. 2011;4:194

24. Tong QB, Chen R, Zhang Y, Yang GJ, Kumagai T, Furushima-Shimogawara R, et al. A new surveillance and response tool: risk map of infected Oncomelania hupensis detected by Loop-mediated isothermal amplification (LAMP) from pooled samples. Acta Trop. 2015;141:170-7.

25. Posthuma-Trumpie GA, Korf J, van Amerongen A. Lateral flow (immuno) assay: its strengths, weaknesses, opportunities and threats. A literature survey. Anal Bioanal Chem. 2009;393:569-82.

26. Jaroenram W, Owens L. Recombinase polymerase amplification combined with a lateral flow dipstick for discriminating between infectious Penaeus stylirostris densovirus and virus-related sequences in shrimp genome. J Virol Methods. 2014;208:144-51.

\section{Submit your next manuscript to BioMed Central and we will help you at every step:}

- We accept pre-submission inquiries

- Our selector tool helps you to find the most relevant journal

- We provide round the clock customer support

- Convenient online submission

- Thorough peer review

- Inclusion in PubMed and all major indexing services

- Maximum visibility for your research

Submit your manuscript at www.biomedcentral.com/submit
Biomed Central 